\title{
Contrast and assimilation in lightness judgments'
}

\author{
JACOB BECK \\ HARVARD UNIVERSITY
}

Experiments studied how assimilation and contrast varied as a function of the reflectance of figures above and below the reflectance of a gray background. The results showed that for the experimental conditions investigated: (a) contrast always occurs when the reflectance of lines is above the reflectance of the background, (b) assimilation occurs when the reflectance is below that of the background, (c) circles produce the same degree of assimilation and contrast as lines of equal width, and (d) repeated judgments do not affect contrast but reduce assimilation; as line darkness and line width increase assimilation gives way to contrast following repeated judgments. The results are discussed in connection with the hypothesis that assimilation and contrast arise from opponent processes in the visual system.

Helson (1964) investigated the changes produced in the lightness of a gray background by the presence of black and white lines. He found that lightness changes went from assimilation (the darkening of the gray background by the black lines or the lightening of it by white lines) to contrast (the darkening of the gray by white lines or the lightening of it by black lines) as a function of line width, line separation, and the reflectance of the background. This finding suggested that assimilation and contrast arise from opponent processes that depend on the differences in stimulation in contiguous areas. Helson hypothesized that small differences in stimulation in neighboring areas result in summation, producing assimilation, and that large differences result in inhibition, producing contrast.

The purpose of the present study was to investigate the phenomena of assimilation and contrast as a function of 'se reflectance of figures above and below the reflectance of a gray background. Three experiments were performed to examine whether the size of the difference in stimulation alone does in fact determine if assimilation or contrast will occur.

\section{EXPERIMENTS I AND II}

Experiments I and II compare the lightness judgments of a gray background when the reflectance of lines and circles was varied:

\section{Method}

The targets in Experiment I consisted of $2 \times 2$ in. gray papers reflecting 20 percent on which vertical lines of varying reflectance were placed. The reflectances of the lines were : 88, 58,49,31, 20, 16, 10, 7 and 5 percent. The lines were separated by $1 / 8$ in. The standard consisted of a gray paper reflecting 20 percent on which seven $1 / 8 \mathrm{in}$. lines of the same gray were over- laid. A tenth target consisting of $1 / 8 \mathrm{in}$. lines reflecting 16 percent on a background reflecting 16 percent was included. This target allowed Os' judgments on a rating scale to be related to an objectively defined measure. A gray of 20 percent reflectance corresponds to a Munsell value of 5 and a gray of 16 percent corresponds to a Munsell value of 4.5. The targets used in Experiment II were identical to those used in Experiment I in all respects except that instead of lines they contained seven rows and columns of circles $1 / 8$ in. in diameter. The distance between the borders of the circles was $1 / 8$ in.

The experiments were mun in a darkroom. The standard and comparison targets were placed against a black background 66 in. from the observers. The incident illumination was $78 \mathrm{ft} .-\mathrm{c}$. The comparison targets were presented in a different irregular order to each O; they were placed randomly to the left or right of the standard. The Os viewed the targets with binocular vision for approximately $3 \mathrm{sec}$. They were instructed to compare the background of the targets and to indicate: (a) which of the two was darker, and (b) the degree of difference, by means of a 7-point rating scale, in which 0 stands for equal lightness; 1 minute difference; 2 very small; 3 small; 4 medium; 5 large; and 6 very large. The scale was typed on a card and placed in front of the Os. The Os made four ratings of each of the targets. The mean of the four ratings was taken as representative of an $O^{\prime}$ 's judgment. To familiarize the Os with the targets, the entire set was presented in a preliminary trial, during which no data were collected. Fifteen undergraduates at Harvard University served in each experiment. The Os were paid to participate and were naive as to the purpose of the experiments.

\section{Results}

Figure 1 presents the relationship between Os'lightness judgments and the reflectance of the lines and circles. The results show that the lines and circles produce similar effects as a function of their reflectance. The mean judgments of the physically darker targets reflecting 16 percent are shown in Fig. 1 by the two unconnected points. The judged lightness of these targets was approximately equal to the darkening produced by assimilation when the reflectance of the lines and circles was 10 percent or less. Of special interest is the fact that the data show a marked asymmetry in the effect produced by figures darker and lighter than the background. Contrast occurred when the reflectance of the lines and circles was above the reflectance of the background and assimilation occurred when the reflectance was below that of the background. Thus, targets 


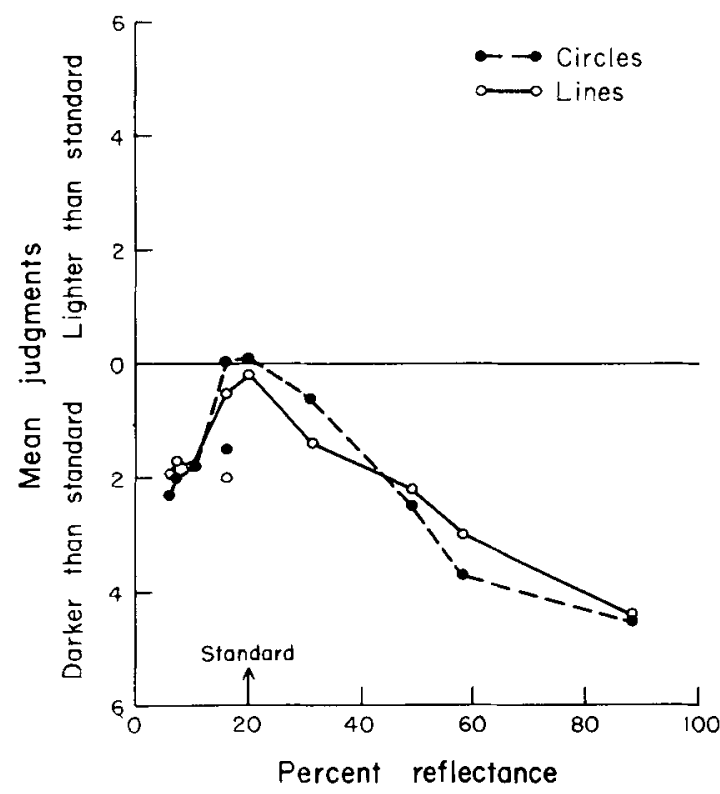

Fig. 1. Mean líghtness judgments of a gray background (reflectance 20 per cent) as a function of the reflectance of overlying lines and circles. The unconnected points represent the mean lightness judgments of objectively darker targets (reflectance 16 per cent) overlaid by lines and circles reflecting 16 per cent. The separation between figures was $1 / 8$ in.

with figures bearing similar ratio relationships to the gray of the background (reflectances of 7 and 58 percent) darkened the gray through assimilation in the first case, and through contrast in the second. It should also be noted that the effect of contrast with increasing reflectance is more marked than is the effect of assimilation when reflectance is decreased. The results indicate, therefore, that for the conditions of line spacing and width investigated, whether contrast or assimilation occur depends on whether the figures are lighter or darker than the background and not on the size of the difference in stimulation.

\section{EXPERIMENT III}

Experiment III studied the effects of repeated judgments on assimilation and contrast when (a) the reflectance of lines was varied, and (b) the width of black and white lines was varied. This experiment also provided a further check upon the finding that assimilation is more readily produced by lines darker than the background, and contrast is more readily produced by lines lighter than the background.

\section{Method}

To evaluate the effects of repetition, five os made four judgments of each of 21 targets on four alternate days. The targets consisted of the ten targets used in Experiment I. An additional target, which consisted of vertical lines $1 / 8 \mathrm{in}$. wide, reflecting 2 percent, was included. Ten further targets were added consisting of
$2.5 \times 2.5$ in. gray papers reflecting 20 percent, on which were placed vertical lines of varying widths. The widths of the lines were: $1 / 16,1 / 8,1 / 4,1 / 2$, and 1 in. The lines were separated by $1 / 8$ in. On five of the targets the lines were cut from a white paper reflecting 88 percent, and on five of the targets the lines were cut from a black paper reflecting 7 percent. The standard consisted of a gray paper reflecting 20 percent, on which $1 / 4-$ in. lines of the same reflectance were placed. The procedure was identical to that of Experiments I and II.

\section{Results}

Figure 2 shows the results when the reflectance of the lines was varied. The broken line represents Os' mean judgments in Session 1; the solid line Os' mean judgments in Session 4. As can be seen in Fig. 2, Os' judgments in Session 1 are comparable to those obtained in Experiment I. Lines darker than the background always produced assimilation, and lines lighter than the background always produced contrast. It is notable that black lines reflecting only 2 percent still produced assimilation. Thus, the results confirm the finding that the effects of lines darker and lighter than the background are not similar. The assimilation produced by lines of 10 percent or less reflectance again resulted in a lightness similar to that of the physically darker target reflecting 16 percent.

Examination of Fig. 2 shows that repetition affected Os' lightness judgments when the lines were darker

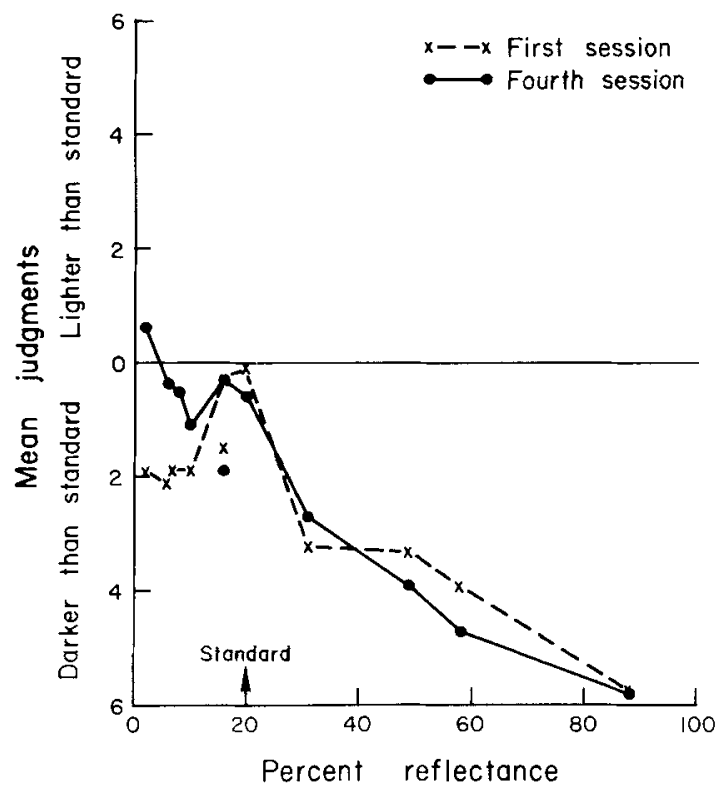

Fig. 2. Effect of repeated judgments on the contrast and assimilation produced on a gray background (reflectance 20 per cent) by overlying lines of varying reflectance. The broken lines represent Os' judgments in Session 1, and the solid line represent os' judgments in Session 4. The unconnected points represent the mean judgments in Sessions 1 and 4 of an objectively darker target (reflectance 16 per cent) overlaid by lines reflecting 16 per cent. The separation between lines was $1 / 8$ in. 
than the background but not when the lines were lighter than the background. The assimilation produced by lines darker than the background was greatly reduced with repeated judgments. In fact, as line darkness increased, assimilation gave way to contrast in Session 4 . An analysis of variance of the difference in Os' lightness judgments in Sessions 1 and 4 of the targets with lines darker than the background is significant at the .025 level. For the targets with lines of greater reflectance than the background, a similar analysis falled to be significant $(p>.25)$.

The results when the width of the lines was varied, presented in Fig. 3, show that assimilation decreases with increasing width of the black lines. An unexpected result is that for the white lines in Session 1 there was no increase in contrast with increasing line width. ${ }^{2}$ As when line reflectance was varied, repeated judgments decreased the effects of assimilation, but did not modify the effect of contrast. Assimilation gives way to contrast as line width increases in Session 4 . An analysis of variance of the difference between Os' lightness judgments of the targets with black lines in Sessions 1 and 4 is significant $(p<.05)$. An analysis of variance of the difference of Os' judgments on Sessions 1 and 4 of the targets with white lines is not significant $(p>.25)$.

\section{DISCUSSION}

Helson (1964) hypothesized that the size of the difference of stimulation in neighboring areas alone determines whether contrast or assimilation will occur. The results of the present experiment indicate that, aside from the size of the difference in the intensity of stimulation, the direction of the intensity difference, and the experience of $O s$ in making judgments, determine whether the target exhibits contrast or assimilation.

The fact that (a) for inexperienced os whether contrast or assimilation occurred depended on whether the figures were darker or lighter than the background, but not the size of the difference, and (b) assimilation but not contrast was affected by repetition, suggests that assimilation and contrast, at least in part, involve different processes. It has been established that contrast effects result from neural inhibitory interactions (Graham, 1965). The origins of assimilation, however, are not well understood. One explanation of the present results is that assimilation results from changes in the proximal stimulus due to the scanning of the target. Several observers remarked that when they stared at the pattern with black lines, assimilation was markedly reduced. This report is consistent with the finding of Burnham (1953) that casual observation without rigid fixation facilitates assimilation. The similarity of the results obtained when using lines and circles, however, indicates that if the pattern of fixations is an important variable, lines and circles did not affect it sufficiently

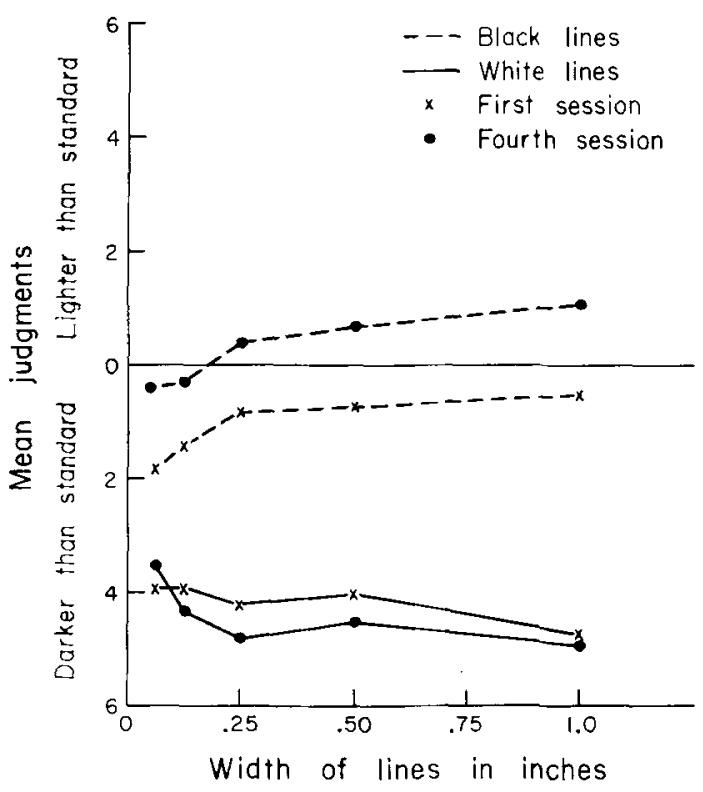

Fig. 3. Effect of repeated judgments on contrast and assimilation produced on a gray background (reflectance 20 per cent) as a function of the width of overlying black lines (reflectance 7 per cent) and white lines (reflectance 88 per cent). The separation between lines was $1 / 8$ in.

to influence the results in a significant way. Whatever factors are involved in assimilation, the present results indicate that for the present experimental conditions when the reflectance of figures is above that of the background, the predominance of inhibition will give rise to contrast. Only when the reflectance of the figures is below that of the background, and inhibition effects are weak, will factors producing assimilation predominate.

\section{References}

Burnham, R. W. Bezold's Color-Mixture Effect. Amer. J. Psychol., $1953,66,377-385$.

Graham, C. H. (Ed.) Vision and visual perception. New York: Wiley, 1965. Pp. 240-246.

Helson, H. Adaptation-level theory. New York: Harper and Row. 1964. Pp. 282-292.

\section{Notes}

1. This research was supported by NSF Grant GB-2901 to the Psychological Laboratories, Harvard University, and by a Grant from the Carnegie Corporation to the Center for Cognitive Studies, Harvard University.

2. In an experiment not reported in this paper, using the set of targets with black and white lines of varying widths used in Experiment III, contrast increased with increasing width of the white lines, and assimilation decreased with increasing width of the black lines.

(Received for publication August 5. 1966.) 